\title{
FUNCTIONAL EQUATIONS INVOLVING MEANS
}

\author{
ZOLTÁN DARÓCZY, KÁROLY LAJKÓ, REZSÔ L. LOVAS, GYULA MAKSA, AND ZSOLT PÁLES
}

ABSTRACT. In this paper, the functional equation

$$
f(p x+(1-p) y)+f((1-p) x+p y)=f(x)+f(y), \quad(x, y \in I)
$$

is considered, where $0<p<1$ is a fixed parameter and $f: I \rightarrow \mathbb{R}$ is an unknown function. The equivalence of this and Jensen's functional equation is completely characterized in terms of the algebraic properties of the parameter $p$. As an application, solutions of certain functional equations involving four weighted arithmetic means are also determined.

\section{INTRODUCTION}

Let $0<p<1$ be fixed. Consider the functional equation

$$
f(p x+(1-p) y)+f((1-p) x+p y)=f(x)+f(y),
$$

where $f: I \rightarrow \mathbb{R}$ is unknown, $I \subset \mathbb{R}$ is a non-void open interval, and (1.1) holds for every $x, y \in I$. Let $S_{p}(I)$ denote the class of all solutions $(f: I \rightarrow \mathbb{R})$ of equation (1.1). Obviously, $S_{p}(I)=S_{1-p}(I)$. Using the terminology of Székelyhidi's book [7], Lajkó's result [5] on equation (1.1) is the following

Theorem 1. $f \in S_{p}(I)$ if and only if there exist symmetric $k$-additive functions $A_{k}: \mathbb{R}^{k} \rightarrow$ $\mathbb{R}(k=0,1,2)$ with the property

$$
A_{2}(p x,(1-p) x)=0 \quad(x \in \mathbb{R})
$$

such that

$$
f(x)=A_{2}(x, x)+A_{1}(x)+A_{0}
$$

for every $x \in I$.

There is a close connection between the functional equation (1.1) and $p$-Wright convex functions (Maksa - Nikodem-Páles [6], Gilányi-Páles [3], Wright [8]). For a fixed $p \in] 0,1[$, $f: I \rightarrow \mathbb{R}$ is $p$-Wright convex on $I$ if

$$
f(p x+(1-p) y)+f((1-p) x+p y) \leqq f(x)+f(y)
$$

Date: April 9, 2008.

2000 Mathematics Subject Classification. 39B22.

Key words and phrases. p-Wright affine function, Jensen-affine function, Linear functional equation, Biadditive function.

This research was partly supported by the Hungarian Scientific Research Fund (OTKA) Grants T-043080, T-038072 and T-047373. 
holds for every $x, y \in I$. Obviously, if $f: I \rightarrow \mathbb{R}$ and $-f: I \rightarrow \mathbb{R}$ are $p$-Wright convex functions on $I$, then $f \in S_{p}(I)$, thus we may call the solutions of (1.1) p-Wright affine functions on $I$. Due to Gilányi and Páles, we have the following theorem [3, Corollary 2].

Theorem 2. $f \in S_{p}(I)$ if and only if, for every point $\xi \in I$, there is a $\delta>0$ such that ]$\xi-\delta, \xi+\delta[\subset I$, and the restriction $f \mid] \xi-\delta, \xi+\delta\left[\right.$ belongs to the class $S_{p}(] \xi-\delta, \xi+\delta[)$.

The property in Theorem 2 was called localizability by the authors. This theorem states the following: if $f: I \rightarrow \mathbb{R}$ is such that for any $\xi \in I$ there is an open interval in $I$ containing $\xi$ on which $f$ satisfies equation (1.1), then $f$ satisfies (1.1) on the whole $I$.

In a recent paper [2], Daróczy, Maksa, and Páles have studied the following problem: do there exist numbers $0<p<1$ such that there is a symmetric biadditive function $A_{2}$ which is not identically zero and which satisfies (1.2)? They showed the existence of such functions if $p$ is transcendental. They also solved the problem for algebraic numbers of second order.

In this paper we first give the complete solution of this problem. As an application, we determine the solutions of the functional equation

$$
f(\alpha x+(1-\alpha) y)+f((1-\alpha) x+\alpha y)=f(\beta x+(1-\beta) y)+f((1-\beta) x+\beta y)
$$

containing four weighted arithmetic means, which is supposed to hold for every $x, y \in I$, where $f: I \rightarrow \mathbb{R}$ is unknown, and $0<\alpha<1,0<\beta<1, \alpha \notin\{\beta, 1-\beta\}$ are given real numbers.

\section{The STRUCTURE of $p$-WRight AFFine FUnCtions}

We begin by stating the following lemmas.

Lemma 1. If $A_{2}: \mathbb{R}^{2} \rightarrow \mathbb{R}$ is a symmetric biadditive function, not identically zero, and $\alpha$ is an algebraic number such that

$$
A_{2}(\alpha x, x)=0 \quad \text { if } x \in \mathbb{R},
$$

then $-\alpha$ is an algebraic conjugate of $\alpha$.

Proof. We have to show that $-\alpha$ is a root of the defining polynomial of $\alpha$ if $\alpha$ is algebraic. Assume that $\sum_{i=1}^{n} t_{i} \alpha^{i}=0$, where $t_{i} \in \mathbb{Z}, i=0, \ldots, n$, and $t_{n} \neq 0$.

Using induction, first we show that

$$
A_{2}\left(\alpha^{k} x, y\right)=(-1)^{k} A_{2}\left(x, \alpha^{k} y\right)
$$

for any integer $k \geqq 0$ and $x, y \in \mathbb{R}$. For $k=0$ (2.2) holds obviously. Since

$$
\begin{aligned}
0=A_{2}(\alpha(x-y), x-y) & =A_{2}(\alpha x, x)-A_{2}(\alpha y, x)-A_{2}(\alpha x, y)+A_{2}(\alpha y, y) \\
& =-A_{2}(\alpha y, x)-A_{2}(\alpha x, y)
\end{aligned}
$$

for any $x, y \in \mathbb{R}$ by (2.1), therefore

$$
A_{2}(\alpha x, y)=(-1) A_{2}(x, \alpha y)
$$

thus (2.2) holds for $k=1$ as well. Suppose that (2.2) holds for a $k \geqq 1$. Then

$$
\begin{aligned}
A_{2}\left(\alpha^{k+1} x, y\right) & =A_{2}\left(\alpha \alpha^{k} x, y\right)=-A_{2}\left(\alpha^{k} x, \alpha y\right) \\
& =(-1)(-1)^{k} A_{2}\left(x, \alpha^{k} \alpha y\right)=(-1)^{k+1} A_{2}\left(x, \alpha^{k+1} y\right),
\end{aligned}
$$


i.e., (2.2) holds for $k+1$ as well.

In contrast with our assertion, suppose that

$$
\beta:=\sum_{i=0}^{n} t_{i}(-\alpha)^{i} \neq 0 .
$$

Let $x, y \in \mathbb{R}$ be arbitrary and denote $z:=\frac{y}{\beta}$. Then, by the biadditivity and by (2.2),

$$
\begin{aligned}
0 & =A_{2}(0, z)=A_{2}\left(\left(\sum_{i=0}^{n} t_{i} \alpha^{i}\right) x, z\right)=\sum_{i=0}^{n} t_{i} A_{2}\left(\alpha^{i} x, z\right) \\
& =\sum_{i=0}^{n} t_{i}(-1)^{i} A_{2}\left(x, \alpha^{i} z\right)=A_{2}\left(x,\left(\sum_{i=0}^{n} t_{i}(-1)^{i} \alpha^{i}\right) z\right)=A_{2}(x, \beta z)=A_{2}(x, y),
\end{aligned}
$$

which is a contradiction, since $A_{2}$ is not identically zero.

Remark. The algebraic numbers $\alpha$ and $-\alpha$ are algebraic conjugates of each other if and only if the defining polynomial of $\alpha$ consists only of terms of even degree.

Lemma 2. There exists a not identically zero symmetric biadditive function $A_{2}: \mathbb{R}^{2} \rightarrow \mathbb{R}$ with the property (2.1) if $\alpha$ is transcendental, or if $\alpha$ is algebraic, and $-\alpha$ is an algebraic conjugate of $\alpha$.

Proof. If both $\alpha$ and $\beta$ are transcendental, by Daróczy's known theorem [1] (see also Kuczma $[4]$ ), there exists an additive function $a: \mathbb{R} \rightarrow \mathbb{R}$, not identically zero, such that

$$
a(\alpha x)=\beta a(x) \quad \text { if } x \in \mathbb{R} .
$$

Also by Daróczy's result [1], if $\alpha$ is algebraic, and $\beta$ is an algebraic conjugate of $\alpha$, then there exists an additive function $a: \mathbb{R} \rightarrow \mathbb{R}$, not identically zero, such that

$$
a(\alpha x)=\beta a(x) \quad \text { if } x \in \mathbb{R} .
$$

Therefore, if $\alpha$ is transcendental, or if $\alpha$ is algebraic, and $-\alpha$ is its algebraic conjugate, then, in both cases, there exists an additive function $a: \mathbb{R} \rightarrow \mathbb{R}$, not identically zero, such that

$$
a(\alpha x)=-\alpha a(x) \quad \text { if } x \in \mathbb{R} .
$$

With this function $a: \mathbb{R} \rightarrow \mathbb{R}$, define

$$
A_{2}(x, y):=a(x) y+a(y) x \quad \text { if } x, y \in \mathbb{R} .
$$

Obviously, $A_{2}: \mathbb{R}^{2} \rightarrow \mathbb{R}$ is a symmetric biadditive function, not identically zero, and

$$
A_{2}(\alpha x, x)=a(\alpha x) x+a(x) \alpha x=-\alpha a(x) x+a(x) \alpha x=0
$$

holds for every $x \in \mathbb{R}$.

In the paper [2] by Daróczy, Maksa and Páles, the following problem was raised.

Problem. Let $0<p<1$ be fixed. When will any $f \in S_{p}(I)$ be a solution of Jensen's functional equation

$$
2 f\left(\frac{x+y}{2}\right)=f(x)+f(y) \quad(x, y \in I) ?
$$


In our next result, we give a complete solution to this problem.

Theorem 3. Let $0<p<1$.

(a) If $\frac{1-p}{p}$ is algebraic, and $-\frac{1-p}{p}$ is not an algebraic conjugate of it, then any element $f \in S_{p}(I)$ is a solution of Jensen's equation (2.3).

(b) If either $\frac{1-p}{p}$ is transcendental, or $\frac{1-p}{p}$ is an algebraic number such that $-\frac{1-p}{p}$ is one of its algebraic conjugates, then there exists a function $f \in S_{p}(I)$ which is not a solution of Jensen's equation (2.3).

Proof. By Theorem 1, it is sufficient to show in case (a) that every symmetric biadditive function $A_{2}: \mathbb{R}^{2} \rightarrow \mathbb{R}$ with the property (1.2) is necessarily identically zero. From (1.2), with the substitution $p x=y$ we obtain

$$
A_{2}\left(\frac{1-p}{p} y, y\right)=0 \quad \text { if } y \in \mathbb{R}
$$

by the symmetry of $A_{2}$. Since $\frac{1-p}{p}$ is algebraic, and $-\frac{1-p}{p}$ is not an algebraic conjugate of it, our assertion follows from Lemma 1.

In case (b), by Lemma 2 , there exists a symmetric biadditive function $A_{2}: \mathbb{R}^{2} \rightarrow \mathbb{R}$, not identically zero, such that

$$
A_{2}\left(\frac{1-p}{p} x, x\right)=0 \quad \text { if } x \in \mathbb{R},
$$

which implies (1.2), thus $x \mapsto A(x, x)(x \in I)$ is not identically zero in (1.3), and this does not satisfy Jensen's equation (see Daróczy - Maksa - Páles [2, Lemma 4.2]).

Theorem 3, together with Theorem 1, gives a complete description of the structure of $p$ Wright affine functions (i.e., functions satisfying equation (1.1)). Thus, every Jensen affine function is $p$-Wright affine, but there are numbers $p$ such that there are $p$-Wright affine functions which are not Jensen affine. These numbers $p$ are characterized as follows: either $p$ is transcendental, or $p$ is algebraic and $\frac{1-p}{p}$ and $-\frac{1-p}{p}$ are algebraic conjugates. It can be shown that, for an algebraic number $p \in] 0,1\left[, p \neq \frac{1}{2}, \frac{1-p}{p}\right.$ and $-\frac{1-p}{p}$ are algebraic conjugates if and only if $p$ and $\frac{p}{2 p-1}$ are algebraic conjugates as well (cf. [3, Theorem 4.3]).

\section{ON A FUNCTIONAL EQUATION INVOLVING FOUR MEANS}

In what follows, we shall study the functional equation (1.5), which involves four means. We may suppose, without loss of generality, that the weights $\alpha$ and $\beta$ satisfy the condition

$$
0<\alpha<\beta \leqq \frac{1}{2}
$$

Let $a<b(a, b \in I)$ be arbitrary, and denote by $P_{a, b}(I)$ the interior of the parallelogram determined by the four points $(a, a),(\alpha a+(1-\alpha) b,(1-\alpha) a+\alpha b),((1-\alpha) a+\alpha b, \alpha a+(1-\alpha) b)$, $(b, b)$. Further, let

$$
P(I):=\bigcup_{a, b \in I, a<b} P_{a, b}(I)
$$


Theorem 4. Suppose that $f: I \rightarrow \mathbb{R}$ satisfies the functional equation (1.5) for every $x, y \in I$, where (3.1) holds. Then, with the notation $\left.p:=\frac{\alpha+\beta-1}{2 \alpha-1} \in\right] 0,1[$, the function $f: I \rightarrow \mathbb{R}$ satisfies the functional equation

$$
f(p u+(1-p) v)+f((1-p) u+p v)=f(u)+f(v)
$$

for every pair $(u, v) \in P(I) \subset I^{2}$, where $P(I)$ is the open subset of $\mathbb{R}^{2}$ defined in (3.2).

Proof. Consider the transformation

$$
\begin{aligned}
& u=\alpha x+(1-\alpha) y \\
& v=(1-\alpha) x+\alpha y
\end{aligned} \quad \text { if }(x, y) \in I^{2},
$$

which takes any point $(x, y) \in I^{2}$ to the point $(u, v) \in I^{2}$. It is easy to see that

$$
\{(u, v)=(\alpha x+(1-\alpha) y,(1-\alpha) x+\alpha y) \mid x, y \in I\}=P(I),
$$

where $P(I)$ is the set defined in (3.2). Solving the system of equations (3.4) for $x$ and $y$, we obtain

$$
\begin{aligned}
& x=\frac{\alpha}{2 \alpha-1} u+\frac{\alpha-1}{2 \alpha-1} v \\
& y=\frac{\alpha-1}{2 \alpha-1} u+\frac{\alpha}{2 \alpha-1} v
\end{aligned}
$$

Thus, by (1.5), we have

$$
\begin{aligned}
f(u)+f(v)= & f\left(\beta\left(\frac{\alpha}{2 \alpha-1} u+\frac{\alpha-1}{2 \alpha-1} v\right)+(1-\beta)\left(\frac{\alpha-1}{2 \alpha-1} u+\frac{\alpha}{2 \alpha-1} v\right)\right) \\
& +f\left((1-\beta)\left(\frac{\alpha}{2 \alpha-1} u+\frac{\alpha-1}{2 \alpha-1} v\right)+\beta\left(\frac{\alpha-1}{2 \alpha-1} u+\frac{\alpha}{2 \alpha-1} v\right)\right) \\
= & f(p u+(1-p) v)+f((1-p) u+p v),
\end{aligned}
$$

hence (3.3) holds indeed.

Theorem 5. If $f: I \rightarrow \mathbb{R}$ satisfies the functional equation (1.5) for any $x, y \in I$, and (3.1) holds, then $f \in S_{p}(I)$, where $\left.p:=\frac{\alpha+\beta-1}{2 \alpha-1} \in\right] 0,1[$.

Proof. By Theorem 4, (3.3) holds for every pair $(u, v) \in P(I) \subset I^{2}$. By Theorem 2, it suffices to show that, for any point $\xi \in I$, there is a $\delta>0$ such that $] \xi-\delta, \xi+\delta[\subset I$, and $f \mid] \xi-\delta, \xi+\delta\left[\in S_{p}(] \xi-\delta, \xi+\delta[)\right.$. Since every point of $\operatorname{diag} I^{2}:=\{(\xi, \xi) \mid \xi \in I\}$ is an interior point of the set $P(I)$, there is a $\delta>0$ for any $\xi \in I$ such that $] \xi-\delta, \xi+\delta\left[^{2} \subset P(I)\right.$, and by (3.3) this means that $f \mid] \xi-\delta, \xi+\delta\left[\in S_{p}(] \xi-\delta, \xi+\delta[)\right.$. Thus we have proved the theorem.

Lemma 3. Let $\alpha, \beta \in] 0,1\left[\right.$ such that $\alpha \notin\{\beta, 1-\beta\}$ and $A_{2}: \mathbb{R}^{2} \rightarrow \mathbb{R}$ a symmetric biadditive function. Then

$$
A_{2}(\alpha x,(1-\alpha) x)=A_{2}(\beta x,(1-\beta) x) \quad(x \in \mathbb{R})
$$

holds if and only if

$$
A_{2}\left(\frac{\alpha+\beta-1}{\alpha-\beta} x, x\right)=0 \quad(x \in \mathbb{R}) .
$$


Proof. Using the symmetry and biadditivity, one can easily check the following identity:

$$
A_{2}((\alpha+\beta-1) y,(\alpha-\beta) y)=A_{2}(\beta y,(1-\beta) y)-A_{2}(\alpha y,(1-\alpha) y)
$$

is satisfied for every $y \in \mathbb{R}$. Therefore (3.5) holds, if and only if

$$
A_{2}((\alpha+\beta-1) y,(\alpha-\beta) y)=0 \quad(y \in \mathbb{R}),
$$

With the substitution $x=(\alpha-\beta) y$, one can immediately see that (3.8) is equivalent to (3.6).

Theorem 6. Let $\alpha, \beta \in] 0,1[$ and $\alpha \notin\{\beta, 1-\beta\}$. The function $f: I \rightarrow \mathbb{R}$ is a solution of the functional equation

$$
f(\alpha x+(1-\alpha) y)+f((1-\alpha) x+\alpha y)=f(\beta x+(1-\beta) y)+f((1-\beta) x+\beta y)
$$

$(x, y \in I)$ if and only if there exist symmetric $k$-additive functions $A_{k}: \mathbb{R}^{k} \rightarrow \mathbb{R}(k=0,1,2)$ with the property

$$
A_{2}(\alpha x,(1-\alpha) x)=A_{2}(\beta x,(1-\beta) x) \quad(x \in \mathbb{R})
$$

such that

$$
f(x)=A_{2}(x, x)+A_{1}(x)+A_{0} \quad \text { if } x \in I .
$$

Proof. $>$ From Theorem 5 it follows that $f: I \rightarrow \mathbb{R}$ is a solution of (1.5) with (3.1) if and only if $f \in S_{p}(I)$, where $\left.p=\frac{\alpha+\beta-1}{2 \alpha-1} \in\right] 0,1[$. By Theorem 1, this is equivalent to the existence of symmetric $k$-additive functions $A_{k}: \mathbb{R}^{k} \rightarrow \mathbb{R}(k=0,1,2)$ with the property

$$
A_{2}(p x,(1-p) x)=0 \quad(x \in \mathbb{R})
$$

such that (3.10) holds. (3.11) holds if and only if

$$
A_{2}\left(\frac{p}{1-p} y, y\right)=0 \quad(y \in \mathbb{R})
$$

i.e.,

$$
A_{2}\left(\frac{\alpha+\beta-1}{2 \alpha-1} \cdot \frac{2 \alpha-1}{\alpha-\beta} y, y\right)=0 \quad(y \in \mathbb{R}) .
$$

This means that (3.6) holds, which is equivalent to (3.9) by Lemma 3.

Now we have again the following question: when is there a solution $f$ of (1.5), such that $x \mapsto A_{2}(x, x)(x \in I)$ is not identically zero in (3.10)? This question is completely answered by the following

Theorem 7. If $\alpha, \beta \in] 0,1[$, and $\alpha \notin\{\beta, 1-\beta\}$, then there is a not identically zero symmetric biadditive function $A_{2}: \mathbb{R}^{2} \rightarrow \mathbb{R}$ satisfying (3.9) if and only if either $\frac{\alpha+\beta-1}{\alpha-\beta}$ is transcendental or it is algebraic such that $-\frac{\alpha+\beta-1}{\alpha-\beta}$ is one of its algebraic conjugates.

Proof. The assertion follows from Lemmas 1, 2 and 3. 
Remarks. 1. Theorems 6 and 7 together characterize the solutions of the functional equation (1.5) involving four weighted arithmetic means. Thus, in (3.10) the term $x \mapsto A_{2}(x, x)$ $(x \in I)$ is identically zero if and only if

$$
t:=\frac{\alpha+\beta-1}{\alpha-\beta} \text { is algebraic, and }-t \text { is not an algebraic conjugate of } t .
$$

In this case, all solutions of the functional equation (1.5) satisfy Jensen's equation (2.3).

2. Denote by $\mathcal{A}(\alpha, \beta)$ the set of not identically zero symmetric biadditive functions $A_{2}$ : $\mathbb{R}^{2} \rightarrow \mathbb{R}$ satisfying $(3.9)(\alpha, \beta \in] 0,1[, \alpha \notin\{\beta, 1-\beta\}$.)

(i) If exactly one of the numbers $\alpha$ and $\beta$ is transcendental, while the other is not $\frac{1}{2}$, then $\frac{\alpha+\beta-1}{\alpha-\beta}$ is transcendental, thus $\mathcal{A}(\alpha, \beta) \neq \emptyset$.

(ii) If either $\alpha$ or $\beta$ is $\frac{1}{2}$, then $\mathcal{A}(\alpha, \beta)=\emptyset$. In this case, (1.5) is equivalent to Jensen's equation $(2.3)$.

(iii) If $a \in\{2 \sqrt{2}, 2 \sqrt[3]{2}\}$, and

$$
\alpha:=\lim _{n \rightarrow \infty}\left(1-\frac{1}{n}\right)^{n}=\frac{1}{e}, \quad \beta:=\frac{a}{a-1}-\frac{a+1}{a-1} \frac{1}{e},
$$

then $\alpha, \beta \in] 0,1\left[, \alpha \notin\{\beta, 1-\beta\}\right.$, and $\frac{\alpha+\beta-1}{\beta-\alpha}=\frac{1}{a}$. Thus $\mathcal{A}(\alpha, \beta) \neq \emptyset$ if $a=2 \sqrt{2}$, but $\mathcal{A}(\alpha, \beta)=\emptyset$ if $a=2 \sqrt[3]{2}$.

\section{REFERENCES}

[1] Z. Daróczy, Notwendige und hinreichende Bedingungen für die Existenz von nichtkonstanten Lösungen linearer Funktionalgleichungen, Acta Sci. Math. Szeged, 22 (1961), 31-41.

[2] Z. Daróczy, Gy. Maksa and Zs. Páles, Functional equations involving means and their Gauss composition, Proc. Amer. Math. Soc., 134 (2006), 521-530.

[3] A. Gilányi and Zs. Páles, On Dinghas-type derivatives and convex functions of higher order, Real Anal. Exchange, 27 (2001/2002), 485-493.

[4] M. Kuczma, An Introduction to the Theory of Functional Equations and Inequalities, Prace Naukowe Universitetu Śląskiego w Katowicach Vol. CDLXXXIX (Państwowe Wydawnictwo Naukowe - Universitet Ślaski, Warszawa-Kraków-Katowice, 1985).

[5] K. Lajkó, On a functional equation of Alsina and García-Roig, Publ. Math. Debrecen, 52 (1998), $507-515$.

[6] Gy. Maksa, K. Nikodem and Zs. Páles, Results on t-Wright convexity, C. R. Math. Rep. Acad. Sci. Canada, 13 (1991), 274-278.

[7] L. Székelyhidi, Convolution type functional equations on topological Abelian groups (World Scientific Publishing Co. Inc., Teaneck, NJ, 1991).

[8] E. M. Wright, An inequality for convex functions, Amer. Math. Monthly, 61 (1954), 620-622.

Institute of Mathematics, University of Debrecen, H-4010 Debrecen, Pf. 12, Hungary E-mail address: \{daroczy, lajko, lovasr,maksa,pales\}@math.klte.hu 